\title{
SPROUTING AND SYNAPSE FORMATION PRODUCED BY CARBOCAINE ${ }^{1}$
}

\author{
M. TAL* AND S. ROTSHENKER $\ddagger^{2}$ \\ Department of Anatomy and Embryology, Hebrew University-Hadassah ${ }^{*}$ Dental and $\ddagger$ Medical Schools, P. O. Box 1172, \\ Jerusalem, Israel
}

Received May 19, 1983; Revised August 1, 1983; Accepted August 19, 1983

\begin{abstract}
Injury to one cutaneous pectoris nerve of the frog induces sprouting of and synapse formation by the contralateral homologous intact nerve. It was previously suggested that axotomy initiates a signal for growth in the cell bodies of the injured motor neurons and that this signal is transferred transneuronally across the spinal cord to intact motor neurons (e.g., Rotshenker, S. (1982) J. Neurosci. 2: 1359-1368). The present study was designed to test the hypothesis that axotomy initiated the signal for growth by depriving neuronal somata of a trophic substance derived from the target muscle. The superficial layer of muscle fibers comprising one cutaneous pectoris muscle, the source of the hypothetical trophic substance, was removed by means of local application of the myotoxic local anesthetic Carbocaine. An increased supernumerary pattern of innervation developed in drug-treated muscles first and in contralateral intact muscles thereafter. These results raise the possibility that target muscle fibers play a role as regulators of a signal for growth in the cell bodies of their innervating motor neurons.
\end{abstract}

Axotomy of the nerve to one cutaneous pectoris muscle of the frog produces sprouting and synapse formation, and thus a polyneuronal pattern of innervation, in the intact homologous muscle on the opposite side (Rotshenker and McMahan, 1976; Rotshenker, 1979; Rotshenker and Reichert, 1980). The application of colchicine to one cutaneous pectoris nerve, in doses that inhibit axonal transport but do not produce degeneration, is similarly followed by contralateral sprouting and synapse formation (Rotshenker, 1982). To account for these phenomena, a transneuronal mechanism for the induction of sprouting was formulated; it was suggested that axotomy and colchicine initiate a signal for growth in the injured and colchicine-treated nerve cell bodies that transferred transneuronally across the spinal cord to the intact motor neurons innervating the opposite muscle (Rotshenker, 1979, 1982). The transneuronal induction of sprouting is thus suggested to be composed of two steps: first, the initiation of a signal for growth in the axotomized nerve cell bodies, and second, the transneuronal transfer of a signal for sprouting to intact motor neurons.

\footnotetext{
${ }^{1}$ We wish to thank Drs. J. Camhi and M. Spira for their most valuable comments during the preparation of the manuscript, and $R$. Cohen for her technical assistance. This work was supported by a grant from the Muscular Dystrophy Association of America.

${ }^{2}$ To whom correspondence should be addressed.
}

The previous experiments (Rotshenker, 1979, 1982) further suggested that one possible mechanism by which axotomy and colchicine could initiate a signal for growth in nerve cell bodies is by depriving neuronal somata of a retrogradely transported trophic substance derived from the muscle target. This working hypothesis is supported by a large body of evidence that attributes trophic influences of target tissues on the cell bodies of their innervating neurons (e.g., Varon and Bunge, 1978). For example, nerve growth factor (NGF), which is a known regulator of vital morphological, biochemical, and electrophysiological properties of sympathetic neurons (Black, 1978; Purves and Lichtman, 1978; Varon and Bunge, 1978; Thoenen and Barde, 1980), is synthetized and released by the iris (Ebendal et al., 1980), taken up by nerve endings, and then retrogradely transported to the somata of the sympathetic neurons that innervate the iris (Schwab, 1977).

To test our working hypothesis that muscle fibers may regulate the growth of their innervating motor neurons at the cell body level, we examined the possibility that the removal of muscle fibers, the source of the hypothetical trophic substance, will be followed by transneuronal sprouting and synapse formation. The results obtained in this study agree well with the possibility that a targetderived trophic substance may play a role in signaling the somata of the motor neurons to initiate growth. 


\section{Materials and Methods}

Frogs (Rana pipiens) 6 to $8 \mathrm{~cm}$ long were used. The animals were kept at room temperature and fed once a week on housefly larvae. Surgery was performed on anesthetized $(0.1 \%$ tricaine methanesulfonate) frogs and controlled visually under a dissecting microscope.

Drug application. Carbocaine (methyl bupivacaine, AB Bofors, $5 \%$ in frog Ringer solution) was applied on top of left cutaneous pectoris muscles. The area exposed to the drug was first sealed off by a ring made of Orabase (medical paste, Squibb) to prevent the leakage of Carbocaine to adjacent muscles. After $15 \mathrm{~min}$ of exposure both Carbocaine and Orabase were removed and the region was washed by Ringer solution.

In some of the control experiments the same surgical procedures were carried out except that Ringer solution, rather than Carbocaine, was applied to the muscles. In another set of control experiments Carbocaine was applied to left cutaneous pectoris nerves at the axilla, remote from the muscle, for $15 \mathrm{~min}$. The application of the drug to the nerve was carried out as previously described for the application of colchicine (Rotshenker, 1982).

The electrophysiological identification of polyneuronally innervated muscle fibers. Endplate potentials (epps) were recorded in single curarized muscle fibers $\left(5 \times 10^{-6} \mathrm{gm} /\right.$ $\mathrm{ml}$, curare in Ringer). Muscle fibers in which multiple epps were elicited by graded intensities of stimulation to the whole nerve were considered polyneuronally innervated. In each muscle 50 to 60 muscle fibers were examined, and their incidence of polyneuronal innervation was defined as the percentage of fibers in which multiple epps were recorded. These values were further used to calculate the average values presented throughout the paper; average \pm SEM ( $n=$ number of muscles tested). The electrophysiological identification of polyneuronally innervated muscle fibers underestimates the extent of supernumerary innervation since some threshholds are quite close to one another and since synapses of low quantal content may be missed (e.g., Rotshenker, 1979). The increase over normal in the percentage of muscle fibers in which multiple epps were recorded is taken as evidence for an increased incidence of polyneuronal innervation resulting from sprouting and synapse formation by motor neurons. The validity of this interpretation has been demonstrated repeatedly in a series of electrophysiological and anatomical studies (Reichert and Rotshenker, 1979; Rotshenker and Reichert, 1980; Rotshenker, 1982; Elizalde et al., 1983; Ring et al., 1983).

Electron mciroscopy. Intact and drug-treated muscles were fixed in $1 \%$ glutaraldehyde in $0.09 \mathrm{M}$ phosphate buffer ( $30 \mathrm{~min})$, postfixed in $1 \%$ osmium tetroxide $(60$ $\mathrm{min})$, dehydrated in ethanol, rinsed in propylene oxide, and embedded in thin wafers of Epon. Neuromuscular junctions were thin sectioned and examined in the electron microscope.

Light microscopy. Intact and drug-treated muscles were fixed in $1 \%$ glutaraldehyde in $0.09 \mathrm{M}$ phosphate buffer, embedded in glycolmethacrylate, and cut into $3-\mu \mathrm{m} \mathrm{sec}-$ tions. Sections were moutned on slides and stained for hematoxylin and eosin.

\section{Results}

Structural changes in Carbocaine-treated cutaneous pectoris muscles. The myotoxic local anesthetic Carbocaine (methyl bupivacaine) was applied topically to the external surface of left cutaneous pectoris muscles. The drug induced the degeneration, and later the regeneration preferentially, of the superficial layer of muscle fibers.

Muscle fiber degeneration (Fig. 2) was clearly evident in the light microscope 1 day after drug application. The extent and duration of the injury varied between muscles. Five to $40 \%$ of the muscle fibers were identified morphologically as damaged during the first week after their
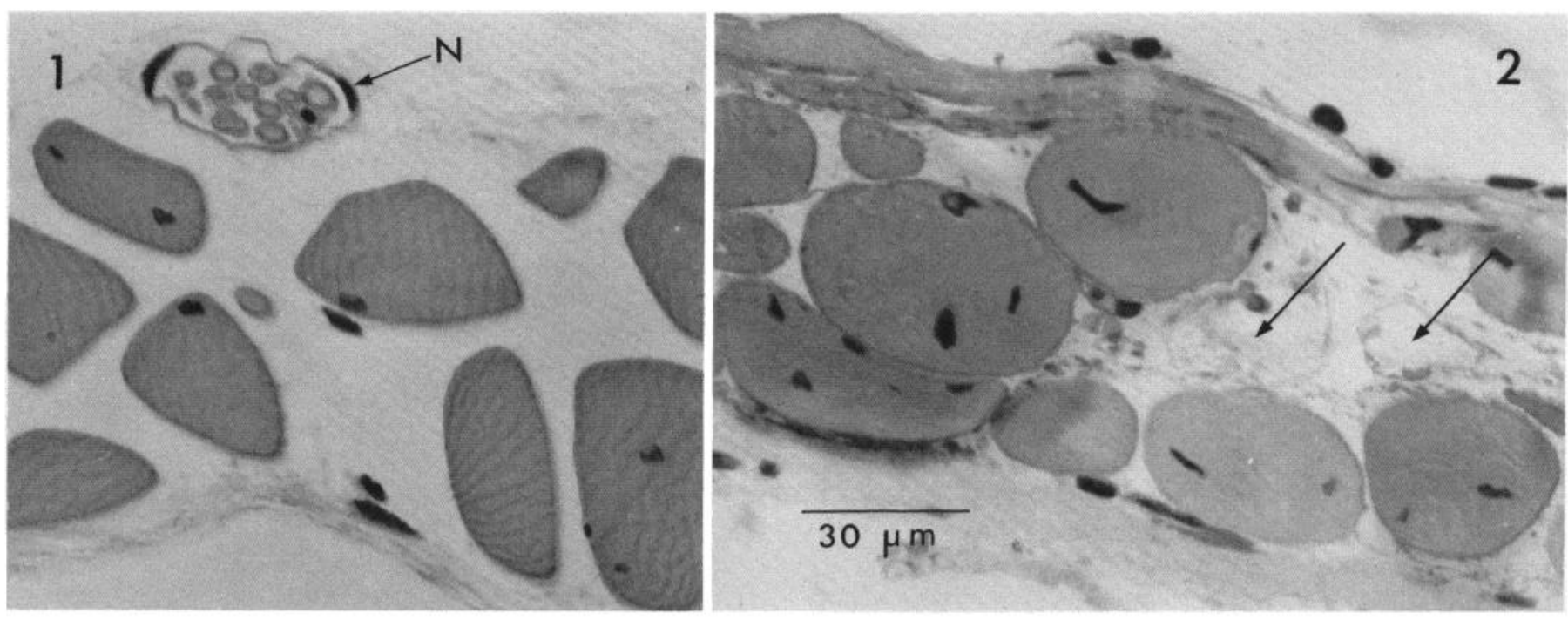

Figure 1. A cross-section through the entire thickness of a normal cutaneous pectoris muscle. $N$, a nerve bundle.

Figure 2. Degenerating muscle fibers and vacated spaces (arrows) previously occupied by muscle fibers 4 days after Carbocaine application. 

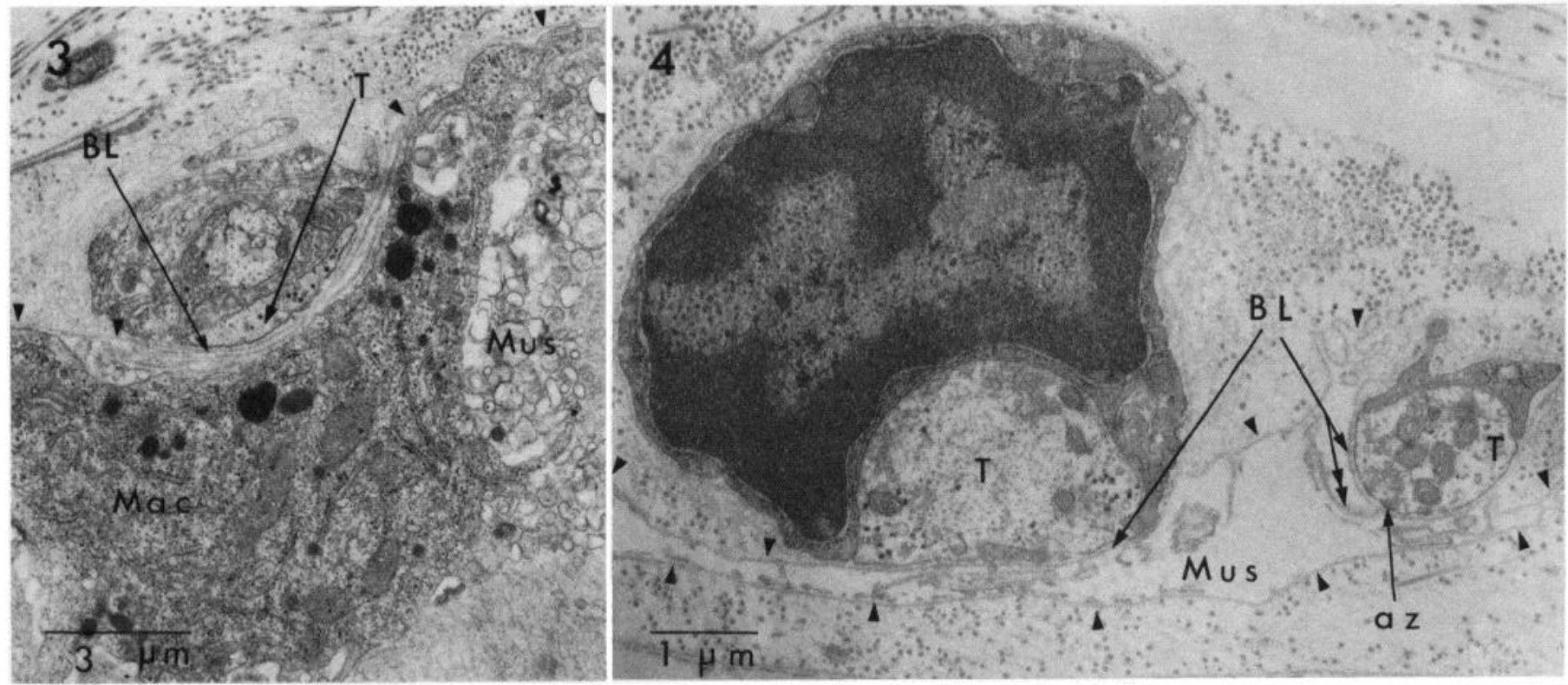

Figure 3. A degenerating muscle fiber (Mus) invaded by a macrophage $(M a c)$. The normal cytoarchitecture of the muscle fiber cannot be identified. The nerve terminal $(T)$ is closely apposed to the synaptic basal sheath $(B L)$ that is continuous with the basal lamina sheath that normally surrounds the muscle fiber (arrowheads).

Figure 4. Two nerve terminals $(T)$ deprived of their target muscle fiber. Their subcellular structure is normal; mitochondria, synaptic vesicles, active zone $(a z)$, neurofilaments, and microtubles are present. The nerve endings retain their close apposition to the junctional basal lamina $(B L)$. The entire lamina sheath (arrowheads) encloses an empty space (Mus) that was created by the phagocytosis of the degenerating muscle fiber. Note the portion of junctional basal lamina (double-head arrow) normally associated with the postsynaptic fold.

exposure to Carbocaine. These values reflect an underestimate of the magnitude of the damage, since they are based on light microscopy that reveals only the most pronounced morphological deviation from normal. It is quite possible that a proportion of muscle fibers was reversibly damaged without undergoing degeneration, and therefore went unnoticed. Degeneration gradually subsided during the next week. Electron microscopy revealed the presence of macrophages that invaded degenerating muscle fibers (Fig. 3) and phagocytized them leaving behind vacated spaces (Fig. 2) enclosed by the basal lamina sheath of the muscle fiber (Fig. 4).

Targetless nerve terminals (Fig. 4) were identified electron microscopically through the period of 3 to 10 days after drug application, which is only a low estimate of the duration for which nerve endings were deprived of their target muscle fibers. Through the entire period sampled, motor axons and nerve endings retained their normal ultrastructure (Fig. 4). Similar observations were also made in mammalian preparations (Jirmanova, 1975). In another study, we have tested some functional aspects of the motor neurons deprived from muscles by studying the ability of the motor neuronal endings to turn over synaptic vesicles. In a quantitative electron microscopic study (Tal and Rotshenker, 1983) it was observed that the spontaneous and stimulation-dependent uptake of horseradish peroxidase (HRP) into normal and target-deprived motor nerve endings were the same through the period of 2 to 8 days after drug application. We thus conclude that the application of Carbocaine leaves the motor nerve terminals intact in terms of ultrastructure and at least certain aspects of function.
Muscle fiber regenerations was first observed 4 days after drug application in the form of dividing myoblasts and myotubes (Fig. 5). Myofibers at early differentiating stages (Fig. 6) were identified as early as 8 days after Carbocaine application. Structural differentiations proceeded for an additional 3 to 4 weeks.

The present results suggest that the muscle fiber degeneration and regeneration induced in the frog skeletal muscle by Carbocaine resemble those that were induced by the drug in mammalian skeletal muscles; but the time course is slower in the frog (e.g., Benoit and Belt, 1970; Jirmanova and Thesleff, 1972; Jirmanova, 1975).

Intact cutaneous pectoris muscles on the side of the body opposite to that exposed to Carbocaine were also examined anatomically. Less than $1 \%$ of the muscle fibers appeared abnormal, usually possessing multiple holes. The same was also observed in intact muscles of unoperated frogs. We did not find any evidence for muscle fiber regeneration as observed in drug-treated muscles. These observations suggest that intact muscles were not exposed to any significnat degree to the Carbocaine that was applied to contralateral muscles.

Supernumerary innervation in cutaneous pectoris muscle following Carbocaine application. Left and right cutaneous pectoris muscle of the same frog were examined electrophysiologically for supernumerary innervation after the application of Carbocaine to left muscles only. The pattern of supernumerary innervation in contralateral right intact muscles was normal for the first 4 weeks, exhibiting an average icidence of polyneuronal innervation of $15.7 \pm 2.3 \%(n=10)$. Thereafter, through days 26 to 165 after drug application, it increased 2-fold over 

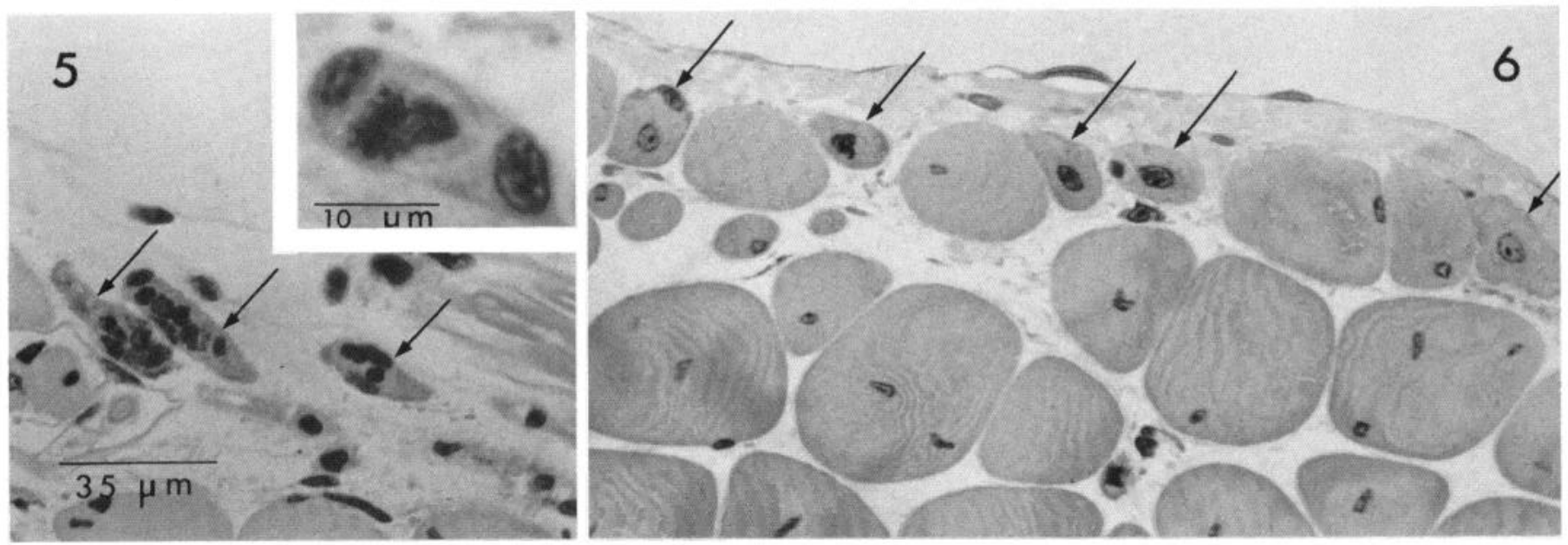

Figure 5. Myotubes (arrows) in a muscle that was exposed to Carbocaine 8 days prior to examination. The myotubes possess a basophilic cytoplasm and multiple dense nuclei which are larger and more oval than those of normal muscle fibers. Inset, A dividing myoblast 4 days after drug application.

Figure 6. Regenerating muscle fibers (arrows) at an early stage of differentiation 9 days after Carbocaine application. The fibers are small in diameter and do not yet show striations as mature muscle fibers do. Their nuclei differ from those of myotubes and mature fibers in that they are centered, large, clear, oval, and contain one to two prominent nucleoli. As differentiation proceeds the young myofibers enlarge in size, their cytoplasm becomes eosinophilic, and striations appear.

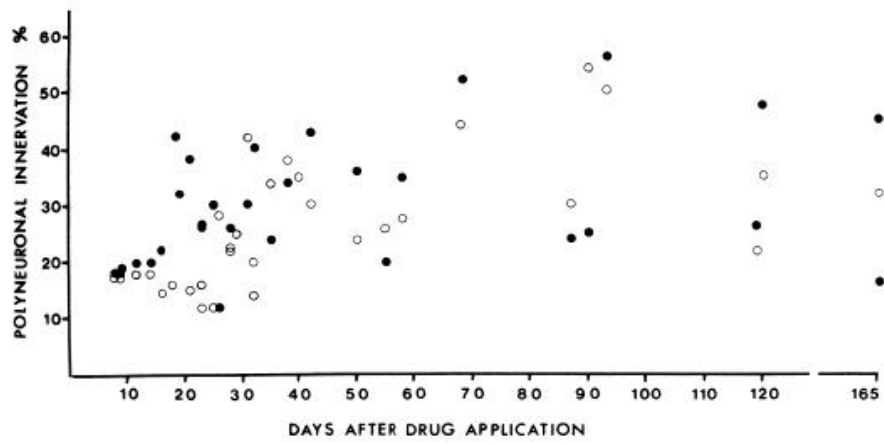

Figure 7. The incidence of polyneuronal innervation in left drug-treated $(O)$ and right intact $(O)$ cutaneous pectoris muscles of the same frogs. A 2 -fold increase in the incidence of polyneuronal innervation developed in the two muscles but with a different timing. The initial increase over normal was first observed at days 18 and 26 after the application of Carbocaine in left and right muscles, respectively.

normal and averaged $31.2 \pm 2.2 \%(n=21)$ (Fig. 7). Thus the exposure of left muscles to Carbocaine is followed, about 4 weeks later, by sprouting and synapse formation and the development of a supernumerary pattern of innervation in contralateral intact right muscles.

Damage to left muscles that were exposed to Carbocaine was mostly confined to the superficial layer of muscle fibers, leaving the inner muscle fiber layer intact (as described above). It was therefore possible to examine the innermost muscle fibers electrophysiologically for supernumerary innervation at any time after the exposure to Carbocaine. The incidence of polyneuronal innervation observed during the first 2.5 weeks after drug application was $19.5 \pm 0.6 \%(n=6)$. The percentage of polyneuronally innervated muscle fibers increased after day 18 and then averaged $32.8 \pm 2.3 \%(n=24)$ through day 165 . Thus additional new synapses developed in left muscles about 2.5 weeks after their exposure to Carbocaine.

Supernumerary innervation that followed Carbocaine application could be secondary to either: (1) the myotoxic effect of the drug and its consequences (as suggested), (2) the surgery and mode of drug application, and (3) the direct effect of the drug on the nerve (e.g., the inhibition of axonal transport or the temporary blockade of impulse activity). To examine the role of surgery and mode of drug application we applied normal Ringer solution to left muscles using the same procedures as those used for Carbocaine application. Left and right muscles that were examined 21 to 124 days after the application of Ringer solution exhibited an incidence of polyneuronal innervation of $18.7 \pm 2.0 \%(n=6)$ and $18.4 \pm 3.3 \%(n$ $=5$ ), respectively. To examine the possible role of Carbocaine on the nerve itself the drug was applied to left cutaneous-pectoris nerves at the axilla remote from the muscle. Left and right muscles were examined 71 days to 98 days after the operation, and the incidence of polyneuronal innervation recorded was $19.5 \pm 1.1 \%(n=$ 6) in left muscles and $18.0 \pm 1.3 \%(n=7)$ in right muscles. These experiments indicate that the supernumerary pattern of innervation observed in left drugtreated and right intact muscles resulted from the myotoxic effects of the drug and its consequences.

\section{Discussion}

Carbocaine that was applied to left cutaneous pectoris muscles produced a polyneuronal pattern of innervation in left treated muscles and contralateral right intact muscles. The drug produced its effect apparently through its myotoxic property and/or one of the consequences that followed: (1) muscle fiber degenration, (2) the removal of muscle fibers, and (3) muscle regeneration. The alternative possibility that Carbocaine induced sprouting 
by damaging axons and nerve endings seems unlikely. As in mammals (e.g., Jirmanova, 1975), the ultrastructure of the motor axons and terminals was not altered from normal by the drug. Furthermore, the uptake of HRP into nerve endings that were deprived of their target muscle fibers by Carbocaine application is undistinguishable from normal 2 to 8 days after drug application (Tal and Rotshenker, 1983). It is still possible, however, that the drug produced some alterations which are below the resolution of our techniques. The present study does not offer any direct evidence that would indicate what is the relative role of either muscle fiber degeneration or the removal of muscle fibers or their regeneration in the initiation of sprouting. However, the results do raise the possibility that target muscle fibers may regulate nerve growth at the neuron's cell body level.

The working hypothesis that was tested in the present study suggests that muscle fibers regulate the growth of the motor neurons innervating them at the level of the nerve cell body; that is, depriving motor neurons of a trophic substance that is transported retrogradely from the muscle initiates in their somata a signal for growth. This proposition is supported by two types of information. First, skeletal muscles are known to synthetize substances that exert trophic influences on spinal motor neurons (e.g., Giller et al., 1977; Brookes et al., 1980; Smith and Appel, 1983). Second, target-derived substances can reach nerve cell bodies and there regulate some of their neuronal properties. An example of the latter is NGF, which is synthetized by the iris (Ebendal et al., 1980) and reaches the cell bodies of sympathetic neurons that innervate the iris. The transport involves the endocytotic activity of the neuronal nerve endings and then the retrograde axonal transport (Schwab, 1977). Depriving these nerve cells of NGF (e.g., by the use of anti-NGF; Nja and Purves, 1978) produced in their cell bodies changes that usually follow axotomy (e.g., chromatolysis and the loss of afferent input). On the other hand, the application of NGF to interrupted axons ( $\mathrm{Nja}$ and Purves, 1978) prevented, to a certain degree, the development of postaxotomy changes.

In the present study, a proportion of muscle fibers were removed by macrophages; other muscle fibers could have been reversibly damaged by the Carbocaine but not to the extent that they underwent complete degeneration. Thus motor neurons became partially deprived of their target cells and possibly also of a muscle-derived trophic substance which might normally be supplied to the nerve cell body through the endocytotic activity of nerve endings and the retrograde axonal transport. The reduction in concentration or amount of the targetderived factor in motor neuronal somata could initiate in them a signal for growth and thus result in sprouting and synapse formation by the same motor neurons onto intact muscle fibers. The signal for growth, if it moves transneuronally across the spinal cord to contralateral intact motor neurons, could induce the recipient motor neurons to sprout and form synapses. But there should be some delay. The results obtained in the present study agree well with this hypothesis. A polyneuronal pattern of innervation developed first in the muscles that were exposed to Carbocaine and then, with a delay of about a week, in contralateral intact muscles. This working hypothesis further agrees with our previous results (Rotshenker, 1979; 1982; Rotshenker and Reichert, 1980) that demonstrate that either axotomy of one cutaneous pectoris nerve or the inhibition of the retrograde axonal transport in it results in sprouting and synapse formation by the contralateral intact cutaneous pectoris motor neurons. All three experimental designs, axotomy, the inhibition of axonal transport, and the removal of muscle fibers, share a common sequella: the interference with the retrograde supply of substances from the periphery of the neuron to its cell body.

Our results thus suggest that a muscle-derived trophic factor may play a role as a central negative regulator of a signal for growth at the cell body level. The absence of this regulator from the neuronal soma appears to induce growth. Such a signal, once elicited, may also be transferred transneuronally to neighboring intact motor neurons. Thus a central mechanism for the initiation of growth and its transneuronal transfer are implicated.

Muscle fiber regeneration could initiate neuronal sprouting via either peripheral or central mechanisms. It has been suggested that developing noninnervated target cells release growth factors that affect the peripheral extensions of neurons and thereby initiate neurite outgrowth. Newly regenerating muscle fibers could conceivably do the same. If such was the case and a diffusable growth factor were to induce sprouting by influencing the peripheral extensions of the motor neuron, then a problem arises regarding the difference in timing between regeneration and sprouting. Myotubes and early differentiating myofibers appeared during the first week after Carbocaine application. However, the earliest we detected an increased pattern of supernumerary innervation in drug-treated muscles and contralateral intact muscles was 18 days and 26 days after Carbocaine application, respectively. Alternatively, regenerating muscle fibers could release a substance that reaches motor neuronal somata where, after a period of accumulation, a growth signal is produced. If such were the case, then the target must play a role as a central positive regulator of the signal for growth.

Muscle fiber degeneration may also play a role in initiating the motor neural sprouting. Again, as in the case of muscle fiber regeneration, one can suggest peripheral and central mechanisms by which degenerative products of muscle fibers may initiate neurite outgrowth. If degenerative products are to affect the peripheral extensions of motor neurons, then again there arises the problem of the relative timing between degeneration and sprouting. If degenerative products are to reach the nerve cell body and thereby initiate a signal for growth, then again a central positive regulatory mechanism for the initiation of the signal for growth is postulated.

\section{References}

Benoit, P. W., and W. D. Belt (1970) Destruction and regeneration of skeletal muscles after local treatment with local anaesthetic bupivacaine. (Marcaine ${ }^{(1)}$ ). J. Anat. 107: 547-556. Black, I. B. (1978) Regulation of autonomic development. Annu. Rev. Neurosci. 1: 183-214. 
Brookes, N., D. R. Burt, A. M. Goldberg, and G. G. Bierkamper (1980) The influence of muscle conditioned medium on cholinergic maturation in spinal cord cell cultures. Brain Res. 186: 474-179.

Ebendal, T., L. Olson, A. Seiger, and K. O. Hedlund (1980) Nerve growth factors in the rat iris. Nature 285: 248-250.

Elizalde, A., M. Huerta, and E. Stefani (1983) Selective reinnervation of twitch and tonic muscle fibers of the frog. J. Physiol. (Lond.) 340: 513-529.

Giller, E. L., J. H. Heale, P. N. Bullock, B. K. Schrier, and P. G. Nelson (1977) Choline acetyltransferase activity of spinal cord cell cultures increased by co-culture with muscle and by muscle conditioned medium. J. Cell Biol. 74: 16-29.

Jirmanova, I. (1975) Ultrastructure of motor end-plates during pharmacologically induced degeneration and subsequent regeneration of skeletal muscle. J. Neurocytol. 4: 141-155.

Jirmanova, I., and S. Thesleff (1972) Ultrastructural study of experimental muscle degeneration and regeneration in the adult rat. Z. Zellforsch. 1.3: 77-97.

Nja, A., and D. Purves (1978) The effects of nerve growth factor and its antiserum on synapses in the superior cervical ganglion of the guina-pig. J. Physiol. (Lond.) 277: 53-75.

Purves, D., and W. J. Lichtman (1978) Formation and maintenance of synaptic connections in autonomic ganglia. Physiol. Rev. 58: 821-862.

Reichert, F., and S. Rotshenker (1979) Motor axon sproutig in intact muscles. Brain Res. 170: 187-189.

Ring, G., F. Reichert, and S. Rotshenker (1983) Sprouting in intact sartorius muscles of the frog following contralateral axotomy. Brain Res. 260: 313-316.

Rotshenker, S. (1979) Synapse formation in intact innervated cutaneous-pectoris muscles of the frog following denervation of the opposite muscle. J. Physiol. (Lond.) 292: 535-547.

Rotshenker, S. (1982) Transneuronal and peripheral mechanisms for the induction of motor neuron sprouting. J. Neurosci. 2: 1359-1368.

Rotshenker, S., and U. J. McMahan (1976) Altered patterns of innervation in frog muscle after denervation. J. Neurocytol. 5: 719-730.

Rotshenker, S., and F. Reichert (1980) Motor axon sprouting and synapse formation in intact innervated skeletal muscle of the frog. J. Comp. Neurol. 193: 413-422.

Schwab, M. E. (1977) Ultrastructural localization of a nerve growth factor-horseradish peroxidase (NGF-HRP) coupling product after retrograde axonal transport in adrenergic neurons. Brain Res. 130: 190-196.

Smith, R. G., and S. H. Appel (1983) Extracts of skeletal muscle increase neurite outgrowth and cholinergic activity of fetal rat spinal motor neurons. Nature 219: 1079-1081.

Tal, M., and S. Rotshenker (1983) Recycling of synaptic vesicles in motor nerve endings separated from their target muscle fibers. Brain Res. 270: 131-133.

Thoenen, H., and Y. A. Barde (1980) The physiology of nerve growth factor. Physiol. Rev. 60: 1248-1335.

Varon, S., and R. Bunge (1978) Trophic mechanisms in the peripheral nervous system. Annu. Rev. Neurosci. 1: 327-361. 